\title{
Impact of prior lamivudine use on the antiviral efficacy and development of resistance to entecavir in chronic hepatitis B patients
}

\author{
Joo An Hwang, Kee Bum Kim, Min Jae Yang, Sun Gyo Lim, Jae Chul Hwang, Jae Youn Cheong, Sung Won Cho, \\ and Soon Sun Kim \\ Department of Gastroenterology, Ajou University School of Medicine, Suwon, Korea
}

Background/Aims: To determine the efficacies of entecavir (ETV) in nucleos(t)ide analogue (NA)-naïve chronic hepatitis $B$ (CHB) patients and in those with prior lamivudine (LAM) use who did not develop resistance.

Methods: We retrospectively enrolled 337 patients with CHB who were treated with ETV ( $0.5 \mathrm{mg}$ daily) for at least 30 months. The study included 270 (80.1\%) NA-naïve patients and 67 (19.9\%) LAM-use patients. Ten of the LAM-use patients were refractory to LAM therapy without developing resistance.

Results: Genotypic resistance to ETV developed more frequently in the LAM-use group (13.1\%) than in the NA-naïve group (2.6\%) at 60 months $(P=0.009)$. In subgroup analysis, after excluding the 10 patients who were refractory to LAM therapy, the cumulative probability of ETV resistance did not differ significantly between the two groups $(P=0.149)$. Prior LAM refractoriness and a higher hepatitis B virus DNA level at month 12 were independent predictive factors for the development of ETV resistance.

Conclusions: ETV resistance developed more frequently in LAM-use patients with CHB. However, prior LAM use without refractoriness did not affect the development of ETV resistance. The serum hepatitis B virus DNA level at month 12 was a major predictor for the development of ETV resistance. (Clin Mol Hepatol 2015;21:131-140)

Keywords: Chronic hepatitis B; Entecavir; Lamivudine; Resistance

\section{INTRODUCTION}

Hepatitis B virus (HBV) infection is a serious public health concern affecting more than 350-400 million people worldwide. Chronically infected people are at risk of HBV-associated complications such as liver cirrhosis, hepatic failure, or hepatocellular carcinoma. ${ }^{2}$ Antiviral therapy for patients with chronic hepatitis B aims to permanently suppress HBV replication, cease the progres- sion of liver damage, and prevent the development of liver-related complications. ${ }^{3}$

Lamivudine (LAM) was used widely as a first-line therapy for chronic hepatitis B during the past decade, mainly due to its antiviral efficacy and safety. ${ }^{4,5}$ However, LAM therapy has a major limitation of developing resistance, which occurs in $15-30 \%$ and $70 \%$ of patients after 1 and 5 years of treatment, respectively. Resistance is usually associated with a rebound in viral load and

\section{Abbreviations:}

ALT, alanine aminotransferase; Cl, confidence interval; ETV, entecavir; $\mathrm{HBeAg}$, hepatitis B e antigen; HBV, hepatitis B virus; HR, hazard ratio; LAM, lamivudine; NA, nucleos(t)ide analogue; PCR, polymerase chain reaction; $V R$, virologic response

\section{Corresponding author : Soon Sun Kim}

Department of Gastroenterology, Ajou University School of Medicine, 164 World cup-ro, Yeongtong-gu, Suwon 443-380, Korea

Tel: +82-31-219-6939, Fax: +82-31-219-5999

E-mail:cocorico99@gmail.com 
often associated with exacerbation of hepatitis. ${ }^{6}$

Entecavir (ETV) is a potent and highly selective inhibitor of HBV DNA polymerase. ${ }^{7}$ Compared to LAM, ETV has been shown to have a high genetic barrier to resistance. The cumulative probability of resistance through 5 years of therapy is reported $1.2 \%{ }^{8}$ Resistance to ETV has been known to occur through a two-hit mechanism. After to initial selection of the rtM204 \pm rtL180 mutations that are responsible for LAM resistance, and additional mutation at rtI169, rtT184, rtS202, or rtM250 is required for ETV resistance. ${ }^{9}$ Therefore, in LAM-resistant patients, switching to ETV is not recommended due to an increased risk of emergence of ETV resistance. ${ }^{10}$ However, data on the antiviral response and development of resistance to ETV in LAM-use patients without developing resistance are limited.

In this study, we compared the efficacy of ETV in nucleos(t)ide analogue (NA)-naïve and LAM-use patients with chronic hepatitis $B$ without the development of resistance and revealed the impact of prior LAM use on the development of ETV resistance.

\section{MATERIALS AND METHODS}

\section{Patients}

Clinical data of patients with chronic hepatitis B treated with ETV $0.5 \mathrm{mg}$ for at least 30 months were retrospectively collected and reviewed. Patients were treated between September 2006 and August 2012 at Ajou University Hospital, Suwon, South Korea. Eligible criteria were: 1) positive hepatitis $B$ surface antigen for at least 6 months before treatment; 2) baseline HBV DNA levels >2,000 IU/mL; and 3) alanine aminotransferase (ALT) levels more than twice the upper limit of normal at the initiation of ETV therapy. Patients who had LAM resistance or experience with previous treatment with a NA other than LAM or evidence of co-infection with hepatitis $C$ were excluded.

A 358 patients with chronic hepatitis B were screened. Twentyone patients did not fulfill the criteria and were excluded from the analysis: six had LAM resistance, six had a baseline HBV DNA level $<2,000 \mathrm{IU} / \mathrm{mL}$, four had a history of treatment with adefovir dipivoxil, or clevudine prior to ETV therapy, respectively, and one patient was co-infected with hepatitis C virus. Therefore, a 337 patients were included in this analysis (Fig. 1).

All patients were tested for Alanine transaminase (ALT), HBeAg, anti-HBe and HBV DNA assessment before and every 3 months during therapy. Virologic response (VR) was defined as serum

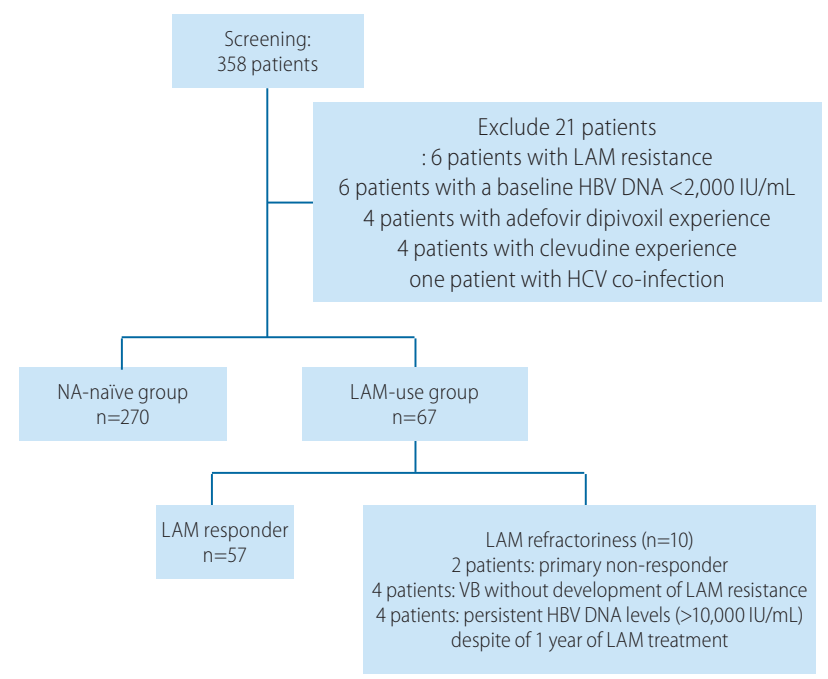

Figure 1. Flow diagram of the study population. LAM, lamivudine; HBV, hepatitis $B$ virus; $N A$, nucleos(t)ide analogue; $H C V$, hepatitis $C$ virus; $V B$, virologic breakthrough.

HBV DNA levels $<20 \mathrm{IU} / \mathrm{mL}$ during the treatment period. Virological breakthrough was defined as an increase in serum HBV DNA level $>1 \log _{10} \mathrm{IU} / \mathrm{mL}$ compared to the nadir HBV DNA level on therapy. ${ }^{3}$ This study protocol was approved by the Institutional Review Board for Human Research at Ajou University Hospital.

\section{Biochemical and serologic testing}

Routine biochemical tests were performed using standard procedures during therapy. Hepatitis B surface antigen, $\mathrm{HBeAg}$, and hepatitis $B$ e antibody were tested using a commercial radioimmunoassay kit (Abbott Laboratories, Chicago, IL, USA). Serum HBV DNA levels were measured using a b-DNA analysis (Versant ${ }^{\mathrm{TM}}$ 3.0, lower limit of detection, 2,000 copies/mL; Bayer Healthcare LLC Diagnostic Division, Tarrytown, NY, USA) from September 2006 to December 2008. Subsequently, serum DNA levels were measured using a quantitative real-time polymerase chain reaction (PCR) assay, COBAS Taq-ManTM HBV test (Roche Diagnostics, Branchburg, NJ, USA) with a lower limit of detection of $20 \mathrm{IU} / \mathrm{mL}$. Samples with HBV DNA levels $<2,000$ copies/mL using the b-DNA assay were retested using the COBAS Taq-Man ${ }^{T M}$ PCR assay. LAM- and ETV-resistant mutations were determined using restriction fragment mass polymorphism technology.

\section{Statistical analysis}

Continuous variables are expressed as mean \pm standard devia- 
tion or median (range) where appropriate. The HBV DNA levels were logarithmically transformed for analysis. Statistical comparisons between two groups were assessed using the Chi-square test and independent t-test where appropriate. Comparisons of means between three groups were analyzed using one way analysis of variance test. Cumulative probabilities of different endpoints were estimated by Kaplan-Meier analysis. Factors associated with the development of ETV resistance were analyzed by Cox regression analyses. All statistical tests are two-sided, and a $P$-value $<0.05$ was considered statistically significant. SPSS version 18.0 was used for all of the statistical analyses (SPSS Inc., Chicago, IL, USA).

\section{RESULTS}

\section{Baseline patient characteristics}

Among 337 patients with chronic hepatitis B, there were 270 (80.1\%) NA-naïve patients (NA-naïve group) and 67 (19.9\%) LAM-use patients (LAM-use group). The mean age of patients were 45.3 (range, 18-69) and 42.7 (range, 23-69) years in the
NA-naïve and LAM-use groups, respectively. The number of patients in the LAM-use group was higher in male than those in the NA-naïve patient group (91.0\% vs. $72.2 \%, P=0.001)$. Proportions of liver cirrhosis and HBeAg positivity were not significantly different between two groups. Mean baseline HBV DNA levels and mean baseline ALT levels were similar in the NA-naïve and LAMuse groups (6.52 \pm 1.05 vs. $6.49 \pm 1.14 \log _{10} \mathrm{IU} / \mathrm{mL}, P=0.816$ and $255.4 \pm 434.2$ vs. $253.1 \pm 256.3 \mathrm{IU} / \mathrm{L}, P=0.967$, respectively). The median ETV administration duration was 42.0 (range 30-60) months in all patients.

LAM-use patients had received prior treatment with LAM for a median duration of 15.0 (range, 2-49) months. Median interval from stopping LAM therapy to initiating ETV therapy was 18.0 (range, 0-108) months. Among the 67 patients who had received prior LAM treatment, 10 were refractory to LAM treatment without the development of LAM resistance. Two patients were primary non-responders to LAM therapy, four patients showed virological breakthrough without the development of LAM resistance, and the rest showed persistent HBV DNA levels $(>10,000 \mathrm{IU} / \mathrm{mL})$ despite 1 year of LAM treatment. These patients showed significantly higher HBV DNA levels at the end of LAM therapy com-

Table 1. Baseline characteristics of the study population

\begin{tabular}{|c|c|c|c|c|}
\hline Factors & $\begin{array}{l}\text { NA-naïve } \\
\text { group } \\
(n=270)\end{array}$ & $\begin{array}{l}\text { LAM-experience } \\
\text { without refractoriness } \\
\text { group }(n=57)\end{array}$ & $\begin{array}{l}\text { LAM-experience with } \\
\text { refractoriness group } \\
\qquad(n=10)\end{array}$ & $P$-value \\
\hline \multicolumn{5}{|l|}{ At start of ETV } \\
\hline Mean age (years, range) & $45.3(18-69)$ & $43.0(23-69)$ & $41.2(26-55)$ & 0.156 \\
\hline Male (n, \%) & $195(72.2)$ & $53(93.0)$ & $8(80.0)$ & 0.001 \\
\hline HBeAg positivity (n, \%) & $141(52.2)$ & $28(49.1)$ & $6(60.0)$ & 0.829 \\
\hline Cirrhosis (n, \%) & $88(32.6)$ & $18(31.6)$ & $0(0.0)$ & 0.366 \\
\hline ALT (IU/L, SD) & $255.4(434.2)$ & $269.3(267.5)$ & $160.8(159.9)$ & 0.737 \\
\hline HBV DNA $\left(\log _{10} 1 \mathrm{U} / \mathrm{mL}, \mathrm{SD}\right)$ & $6.52(1.05)$ & $6.61(1.05)$ & $5.79(1.42)$ & 0.077 \\
\hline Median duration of ETV treatment (months, range) & $42.0(30-60)$ & $42.0(34-60)$ & $45.0(30-60)$ & 0.641 \\
\hline \multicolumn{5}{|l|}{ At start of LAM } \\
\hline HBeAg positivity & & $35(63.6)$ & $9(90.0)$ & 0.148 \\
\hline HBV DNA $\left(\log _{10} \mid \mathrm{U} / \mathrm{mL}, \mathrm{SD}\right)$ & & $6.77(0.98)$ & $6.83(0.82)$ & 0.859 \\
\hline $\begin{array}{l}\text { HBV DNA }\left(\log _{10} I \mathrm{U} / \mathrm{mL}, \mathrm{SD}\right) \text { at the end of LAM } \\
\text { therapy }\end{array}$ & & $3.26(1.55)$ & $5.47(1.29)$ & 0.000 \\
\hline Median duration of LAM treatment (months, range) & & $12.0(2-49)$ & $19.0(8-49)$ & 0.369 \\
\hline $\begin{array}{l}\text { Median interval from LAM treatment to ETV } \\
\text { treatment (months, range) }\end{array}$ & & $22.0(0-108)$ & $0(0-3)$ & 0.000 \\
\hline
\end{tabular}

NA, nucleos(t)ide analogue; LAM, lamivudine; HBeAg, hepatitis B e antigen; ALT, alanine aminotransferase; SD, standard deviation; HBV, hepatitis B virus; ETV, entecavir. 
A

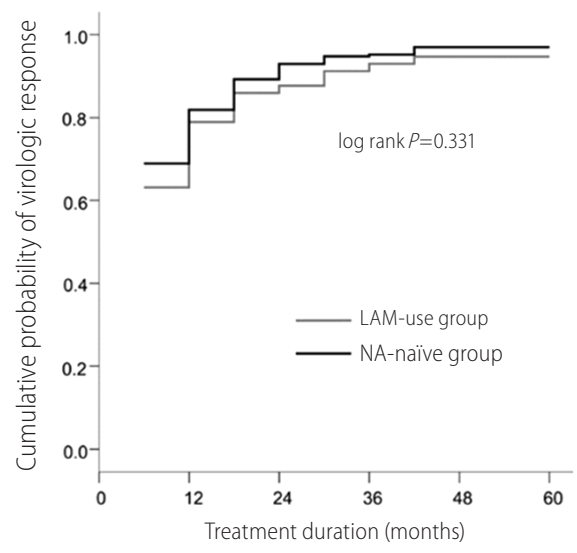

Patients NA-naïve No. at risk LAM-use

$\begin{array}{cccccc}270 & 84 & 29 & 13 & 4 & 1 \\ 67 & 21 & 8 & 5 & 3 & 2\end{array}$

C

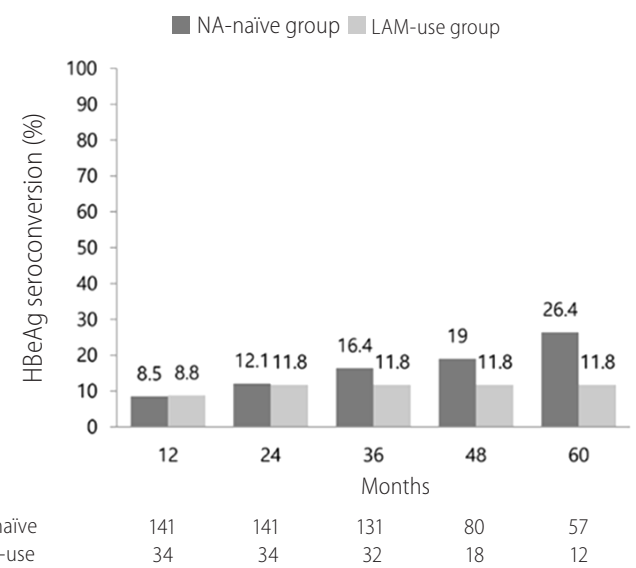

B

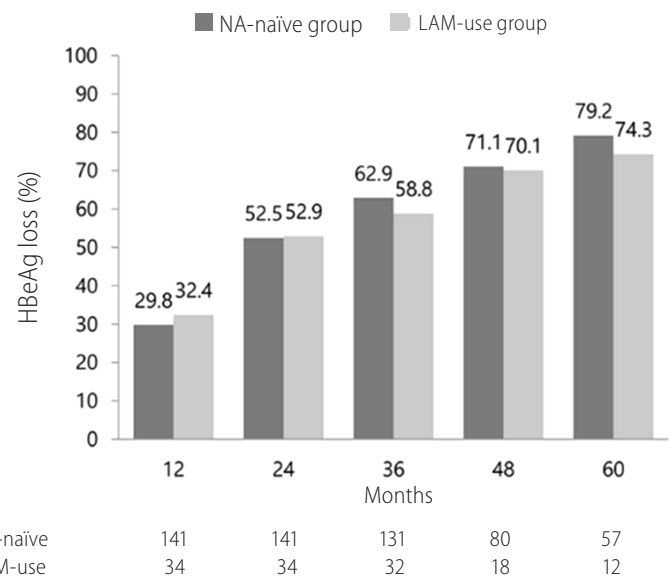

D

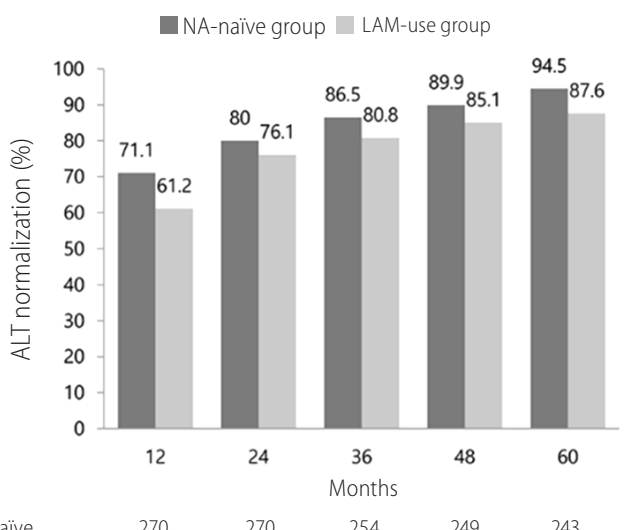

Patients NA-naïve No. at risk LAM-use

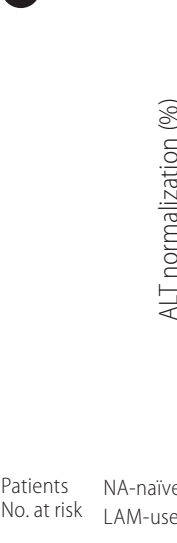

Figure 2. Antiviral efficacies of entecavir (ETV) treatment according to prior lamivudine use in the two study groups. (A) Kaplan-Meier curve for the cumulative probabilities of achieving virologic response. (B) Comparison of the hepatitis B e antigen (HBeAg) loss probabilities during ETV therapy. (C) Comparison of the HBeAg seroconversion probabilities during ETV therapy. (D) Comparison of the ALT normalization probabilities during ETV therapy. LAM, lamivudine; NA, nucleos(t)ide analogue; ALT, alanine aminotransferase.

pared to those without LAM refractoriness. Median interval from stopping LAM therapy to initiating ETV therapy was also significantly shorter in patients with LAM refractoriness compared to those without LAM refractoriness. Baseline characteristics of the study population are presented in Table 1.

\section{Antiviral efficacy of ETV}

Antiviral efficacy of ETV is summarized in Figure 2. The cumulative probabilities of VR are shown in Figure 2A. The VR rates at months $12,24,36,48$, and 60 were $81.9 \%, 93.0 \%, 95.2 \%$, $97.0 \%$, and $97.0 \%$ in the NA-naive group and $79.1 \%, 86.6 \%$, $92.5 \%, 94.0 \%$, and $94.0 \%$ in the LAM-use group, respectively. There was no statistically significant difference between the two groups (log rank $P=0.257)$.

Normalization of ALT at month 60 was observed in $94.5 \%$ and $87.6 \%$ of NA-naïve and LAM-use patients, respectively. There was no statistically significant differences between the two groups (log rank $P=0.201$ ). The cumulative probabilities for biochemical responses at months $12,24,36$, and 48 were $71.1 \%, 80.0 \%, 86.5 \%$, and $89.9 \%$ in the NA-naïve group and $61.2 \%, 76.1 \%, 80.8 \%$, and $85.1 \%$ in LAM-use group, respectively.

Among the 141 patients who were HBeAg-positive in the NAnaïve group, 100 achieved HBeAg loss and 28 patients achieved HBeAg seroconversion during the treatment period. Of the $34 \mathrm{pa}-$ tients who were $\mathrm{HBeAg}$-positive in the LAM-use group, 24 achieved HBeAg loss and four achieved HBeAg seroconversion. The probabilities of HBeAg seroconversion at months 12, 24, 36, 
A

\section{C}

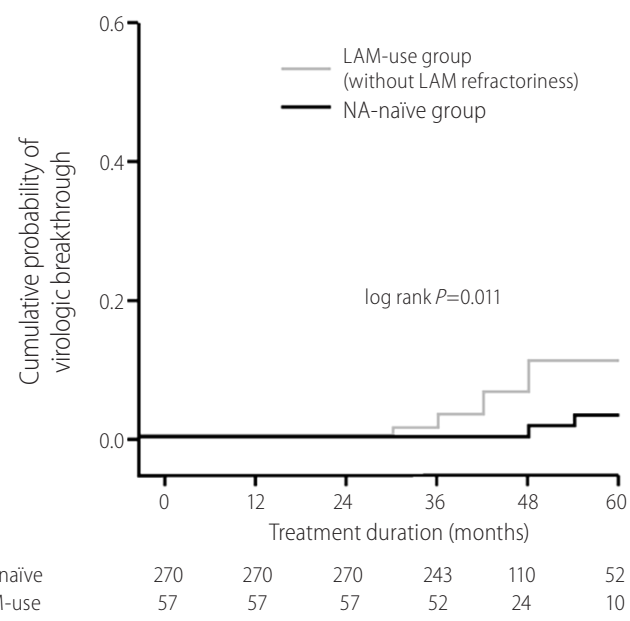

(with/without LAM refractoriness)

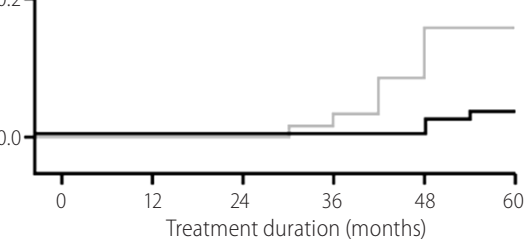

$\log \operatorname{rank} P=0.001$
Patients NA-naïve No. at risk LAM-use
B

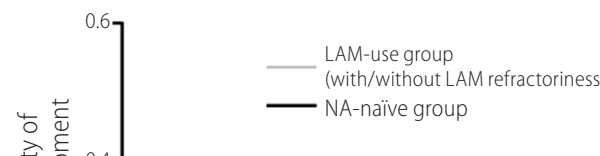

$\log \operatorname{rank} P=0.009$ No. at risk LAM-use
Patients NA-naïve

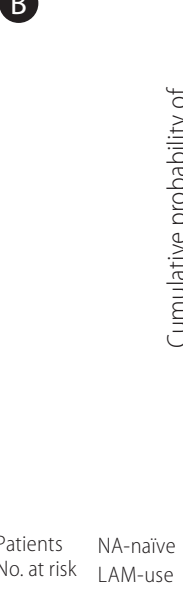

(D)

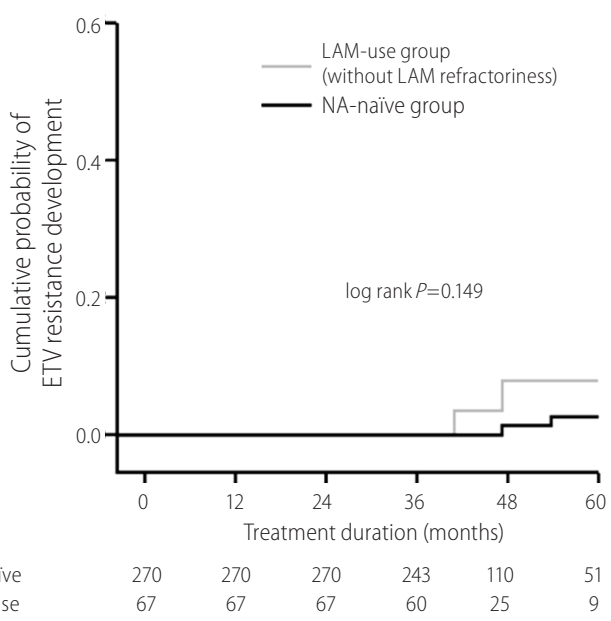

Figure 3. Kaplan-Meier curves for the cumulative probabilities of virological breakthrough and the development of entecavir (ETV) resistance. (A) Comparison of cumulative probabilities of virological breakthrough during ETV treatment between the nucleos(t)ide (NA)-naïve group and lamivudine (LAM)-use group (with or without refractoriness) in all study populations. (B) Comparison of the incidence of ETV resistance between the NA-naïve group and the LAM-use group (with or without refractoriness) in the study population. (C) Comparison of the cumulative probabilities of virological breakthrough during ETV therapy after excluding patients with prior lamivudine experience in the NA-naïve and LAM-use groups. (D) Comparison of the cumulative probabilities of ETV resistance during ETV therapy after excluding patients with prior lamivudine experience in the NA-naïve group and LAM-use group. VB, virologic breakthrough.

48 , and 60 were $8.5 \%, 12.1 \%, 16.4 \%, 19.0 \%$, and $26.6 \%$ in the NA-naïve group and $8.8 \%, 11.8 \%, 11.8 \%, 11.8 \%$, and $11.8 \%$ in the LAM-use group $(P=0.295)$, respectively.

\section{Development of ETV resistance}

During the ETV treatment, virological breakthrough occurred in four patients in the NA-naïve group and six patients in the LAMuse group. The cumulative probabilities of virological breakthrough at months 36,48 , and 60 were $0.4 \%, 2.2 \%$, and $3.5 \%$ in the NA-naïve group, $3.1 \%, 15.8 \%$, and $15.8 \%$ in the LAM-use group. Virological breakthrough occurred more frequently in the LAM-use group than in the NA-naive group (log rank $P=0.001)$ (Fig. 3A). Among the 10 patients who developed virological breakthrough, genotypic ETV resistance was demonstrated in seven (three NA-naive and four LAM-use patients). ETV resistance occurred more frequently in the LAM-use group compared to the NA-naïve group ( $\log$ rank $P=0.009$ ). The cumulative probabilities of ETV resistance at months 36,48 and 60 were $0.4 \%, 1.3 \%$, and $2.6 \%$ in NA-naïve group and $0.0 \%, 13.1 \%$, and $13.1 \%$ in the 
Table 2. Summary of seven patients who developed ETV resistance during therapy

\begin{tabular}{|c|c|c|c|c|c|c|c|}
\hline \multirow{2}{*}{ Patients } & \multicolumn{3}{|c|}{ NA-naïve } & \multicolumn{4}{|c|}{ LAM-use } \\
\hline & 1 & 2 & 3 & 4 & 5 & 6 & 7 \\
\hline Age (years) & 39 & 56 & 50 & 37 & 50 & 30 & 43 \\
\hline Gender & M & M & M & M & M & M & M \\
\hline HBeAg & Positive & Positive & Positive & Positive & Positive & Positive & Positive \\
\hline Duration of LAM treatment (months) & 0 & 0 & 0 & 12 & 12 & 24 & 12 \\
\hline Response to LAM & & & & Responder & Responder & $\begin{array}{l}\text { Virologic break- } \\
\text { through }\end{array}$ & $\begin{array}{l}\text { Primary non- } \\
\text { responder }\end{array}$ \\
\hline Baseline HBV DNA $\left(\log _{10} I \mathrm{I} / \mathrm{mL}\right)$ & 7.24 & 7.24 & 6.89 & 7.24 & 7.24 & 7.24 & 6.95 \\
\hline $\begin{array}{l}\text { Lowest HBV DNA during ETV } \\
\text { treatment (IU/mL, months) }\end{array}$ & $294(26)$ & $<20(24)$ & $<20(24)$ & $235(26)$ & $38(18)$ & $<20(12)$ & $21(37)$ \\
\hline Time to ETV resistance (months) & 30 & 54 & 48 & 48 & 48 & 42 & 42 \\
\hline HBV mutation pattern & $\begin{array}{c}\text { rtL180M + } \\
\text { rtM204V + } \\
\text { rtS202G }\end{array}$ & $\begin{array}{c}\text { rtL180M + } \\
\text { rtM204V } \\
+ \text { rtS202G }\end{array}$ & $\begin{array}{c}\text { rtL180M + } \\
\text { rtM204V + } \\
\text { rtS202G }\end{array}$ & $\begin{array}{c}\text { rtL180M + } \\
\text { rtM204V + } \\
\text { rtT184L }\end{array}$ & $\begin{array}{c}\text { rtL180M + } \\
\text { rtM204V + } \\
\text { rtS202G }\end{array}$ & $\begin{array}{c}\text { rtL180M + } \\
\text { rtM204V + rtT184I/ } \\
A+r t S 202 G\end{array}$ & $\begin{array}{l}\text { rtL180M + } \\
\text { rtM204V + } \\
\text { rtM250V }\end{array}$ \\
\hline Rescue therapy & ETV+ADV & ETV+TDF & ETV+TDF & ETV+ADV & TDF & ETV+ADV & ETV+ADV \\
\hline
\end{tabular}

ETV, entecavir; NA, nucleos(t)ide analogue; LAM, lamivudine; M, male; HBeAg, hepatitis B e antigen; HBV, hepatitis B virus; ADV, adefovir dipivoxil; TDF, tenofovir.

Table 3. Risk factors for genotypic resistance to ETV in patients with chronic hepatitis B

\begin{tabular}{|c|c|c|c|c|}
\hline \multirow{2}{*}{ Factors } & \multicolumn{2}{|c|}{ Univariate analysis } & \multicolumn{2}{|c|}{ Multivariate analysis } \\
\hline & $\mathrm{HR}(95 \% \mathrm{Cl})$ & $P$-value & $\mathrm{HR}(95 \% \mathrm{CI})$ & $P$-value \\
\hline Age ( $\geq 50$ years vs. $<50$ years) & $1.47(0.33-6.58)$ & 0.613 & & \\
\hline Male sex & $30.73(0.02-51425.70)$ & 0.366 & & \\
\hline HBeAg positivity & $51.54(0.11-24379.19)$ & 0.213 & & \\
\hline Baseline ALT & $1.00(0.99-1.00)$ & 0.727 & & \\
\hline Baseline HBV DNA $\left(\log _{10} 1 \mathrm{U} / \mathrm{mL}\right)$ & $2.80(0.67-11.64)$ & 0.158 & & \\
\hline Presence of liver cirrhosis & $0.02(0.00-13.88)$ & 0.249 & & \\
\hline LAM use without refractoriness (vs. naïve) & $3.45(0.57-20.60)$ & 0.176 & & \\
\hline $\begin{array}{l}\text { LAM refractoriness (vs. LAM use } \\
\text { without refractoriness or naïve) }\end{array}$ & $18.46(3.08-110.73)$ & 0.001 & $9.00(1.43-56.64)$ & 0.019 \\
\hline Duration of ETV treatment & $1.03(0.91-1.15)$ & 0.670 & & \\
\hline HBV DNA at month 12 ( $\geq 250 \mathrm{vs} .<250 \mathrm{IU} / \mathrm{mL}$ ) & $13.91(2.68-72.14)$ & 0.002 & $10.36(1.91-56.32)$ & 0.007 \\
\hline
\end{tabular}

ETV, entecavir; HR, hazard ration; Cl, confidence interval; HBeAg, hepatitis B e antigen; ALT, alanine aminotransferase; HBV, hepatitis B virus; LAM, lamivudine.

LAM-use group (Fig. 3B).

Table 2 summarizes the characteristics of the seven patients who developed ETV resistance during therapy. All patients who developed ETV resistance were male and $\mathrm{HBeAg}$-positive. The median baseline HBV DNA level was $7.24 \log _{10} \mathrm{IU} / \mathrm{mL}$ and the median time to developing a mutation was 48.0 (30-54) months. Four patients with LAM use had received prior treatment with LAM for a median duration of 12.0 (12-24) months. Among these patients, two showed refractory responses to prior LAM therapy. The most common ETV-resistant mutation was rtS202G variant (four patients), while others had rtM250V, rtT184L, and rtS202G + rtT184I/A variants, respectively. Patients were added adefovir dipivoxil or tenofovir to ETV or changed to tenofovir monotherapy after ETV resistance was detected.

\section{Subgroup analysis excluding patients who were refractory to LAM treatment}

Patients with prior LAM use without the development of resistance can be divided into two groups: LAM responders and LAM- 


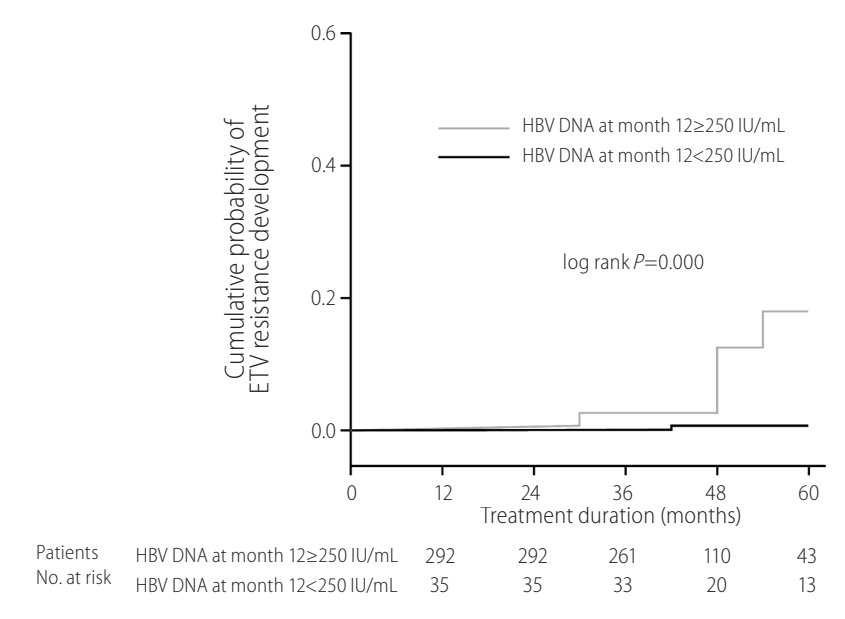

Figure 4. Kaplan-Meier curves for the cumulative probabilities of the development of entecavir (ETV) resistance according to serum hepatitis $B$ virus (HBV) DNA level at 12 months. Patients with a higher HBV DNA level ( $\geq 250 \mathrm{IJ} / \mathrm{mL}$ ) at 12 months of ETV therapy were more likely to develop ETV resistance.

refractory subjects. We assumed that LAM-refractory patients might have higher probabilities for developing ETV resistance than LAM responders, even if they showed no LAM-resistant mutations at baseline. Therefore, subgroup analysis for the development of ETV resistance was done after excluding 10 patients who were refractory to LAM therapy.

Virological breakthrough occurred still frequently in the LAMuse group than NA-naïve group (log rank $P=0.011$ ) (Fig. 3C). However, ETV resistance occurred similarly in both LAM-use and NA-naïve group (log rank $P=0.149$ ). The cumulative probabilities of ETV resistance at months 36,48 , and 60 were $0.4 \%, 1.3 \%$, and $2.6 \%$ in the NA-naïve group and $0.0 \%, 7.9 \%$, and $7.9 \%$ in the LAM-use group, respectively (Fig. 3D).

\section{Predictive factors for ETV resistance}

Baseline characteristics and serum HBV DNA levels at month 12 were analyzed to identify the predictive factors for the development of ETV resistance. On univariate and subsequent multivariate analysis of all study patients, high HBV DNA level ( $\geq 250 \mathrm{IU} /$ $\mathrm{mL}$ ) at 12 months of ETV treatment and previous LAM refractoriness were independent predictive factors for developing ETV resistance (Hazard ratio [HR], 10.36; 95\% confidence interval [CI], 1.91-56.32; $P=0.007$ and $\mathrm{HR}, 9.00 ; 95 \% \mathrm{Cl}, 1.43-56.64$; $P=0.019$, respectively) (Table 3, Fig. 4). Previous LAM use without refractoriness did not affect the development of ETV resistance. Baseline HBV DNA level, HBeAg positivity, and the presence of liver cirrhosis were also statistically insignificant factors for predicting ETV resistance.

\section{DISCUSSION}

The present study revealed the comparable antiviral efficacy of ETV in LAM-use patients to NA-naïve patients with chronic hepatitis $B$ and also newly discovered similar probabilities of ETV resistance development in two groups except patients with prior LAM refractoriness.

The present study explored the efficacy of ETV in NA-naïve and LAM-use patients with chronic hepatitis B who did not develop LAM resistance. The antiviral effect of ETV in NA-naïve patients in our study coincided with the results demonstrated in the large phase III registration trials. ${ }^{7,11}$ Furthermore, in this study, cumulative VR rates were not significantly different between the NA-naive and LAM-use groups. This result is consistent with those of a previous study that reported that LAM use without the development of LAM resistance does not influence the antiviral influence of ETV. ${ }^{12,13}$ However, these studies included only small number of patients with LAM use without resistance $(n=20-33)$ and followed patients up for a very short duration (median duration of 11 and 24 months, respectively). Therefore, the present study supported the previous findings with convincing evidence. On the other hand, a recent study reported that a lower probability of achievement of VR in LAM-use patients without LAM resistance than in NA-naive patients treated with ETV. ${ }^{14}$ In this study, the LAM-use group was largely composed of patients who experienced virological breakthroughs under LAM therapy (32.6\%). This difference can be a reason for the discordance. Meanwhile, cumulative probabilities of HBeAg loss in the present study seemed to be higher than previous study. Previous study reported that cumulative rate of HBeAg loss reach about $42 \%$ at 4 year of ETV treatment in NA-naïve patients. ${ }^{15}$

We also evaluated the impact of prior LAM use on the development of ETV resistance. The possibility of a virological breakthrough was increased in the LAM-use group compared to the NA-naïve group ( $\log$ rank $P=0.001)$. Furthermore, genotypic ETV resistance was developed more frequently in the LAM-use patients ( $\log \operatorname{rank} P=0.009$ ). The probability of ETV resistance in the NA-naïve group was comparable to the results of a previous study. ${ }^{8}$ The great likelihood of occurring ETV resistance in the LAM-use group seemed to be in accordance with the findings of a recent study. ${ }^{14}$ However, subgroup analyses excluding patients 
who were refractory to LAM therapy without developing LAM resistance showed no statistical difference between the two ETV resistance groups. Cox regression analysis also demonstrated that LAM use without refractoriness is not a risk factor for the development of ETV resistance. However, LAM refractoriness was a significant risk factor for the development of ETV resistance (HR, 9.00; $95 \% \mathrm{Cl}, 1.43-56.64 ; P=0.019$ ). Therefore, we assumed that higher probability of the development of ETV resistance in a recent study is not due to LAM use but LAM refractoriness, which might accompany an undetectable portion of mutant HBV. A recent study reported that cumulative probabilities of ETV resistance development were $0.0 \%, 8 \%$, and $28 \%$ at month 48 in NA-naïve group, the LAM-exposed group without resistance, and the LAM resistance group, respectively. However, subgroup analysis showed that lower probability of ETV resistance development (1.9\% at month 48$)$ in patients who had achieved complete virologic suppression for more than 1 year with prior LAM treatment. ${ }^{14}$ Furthermore, a Japanese study reported that switching from LAM to ETV therapy in stable patients resulted in increased rates of virologic suppression with no evidence of ETV resistance through 2 years of therapy. ${ }^{13}$ Another Japanese study reported that the efficacy of ETV after switching from LAM is the highest in patients with no rtM204V/I substitutions and a viral load $<5 \log _{10}$ copies $/ \mathrm{mL}{ }^{16}$ Therefore, the response to LAM treatment must be considered in the evaluation of ETV therapy for the treatment of chronic hepatitis B in patients with prior LAM use.

On the other hand, virological breakthrough was more frequently observed in patients with LAM use without refractoriness than NA-naïve patients ( $\log$ rank $P=0.011)$. This discrepancy between development of virological breakthrough and ETV resistance is uncertain. All patients who developed virological breakthrough without ETV resistance were treated with tenofovir as a rescue therapy. Therefore, it was not possible to confirm the later development of ETV resistance in these patients.

The present study also revealed the risk factors for the development of genotypic resistance to ETV. A serum HBV DNA >250 IU/ $\mathrm{mL}$ at 12 month and LAM refractoriness were the independent risk factors for the development of ETV resistance. There was no well-known risk factor for development of ETV resistance except prior LAM resistance because ETV resistance rarely develops in NA-naïve patients. Meanwhile, VR at 24 week during treatment with LAM or telbivudine and at 48 week during treatment with adefovir dipivoxil is associated with a lower incidence of resistance. ${ }^{17-20}$ Therefore, current guideline recommends changing to a more potent drug in patients receiving LAM, telbivudine, or ade- fovir dipivoxil with a partial virologic response at week 24 or 48 , respectively. ${ }^{3}$ However, the optimal management of patients with partial virologic response under ETV is currently debatable. The vast majority of patients with a partial virologic response to ETV therapy achieved complete virologic response through prolonged ETV therapy with a very low chance of viral resistance. ${ }^{21,22}$ Therefore, careful inspection for the development of ETV resistance is required in patients with higher HBV DNA levels after 1 year of ETV treatment, as the common strategy of changing to other potent drugs such as tenofovir cannot be applied uniformly.

Tenofovir is another very potent NA with a high barrier to resistance that is recommended as the first-line monotherapy in patients with chronic hepatitis B. ${ }^{3}$ Although only short-term clinical data are available for tenofovir, the VR among LAM-use or -resistant patients who received tenofovir monotherapy was not significantly different from those of treatment-naïve patients without the development of tenofovir resistance. ${ }^{23-25}$ Therefore, tenofovir can be a preferred treatment option for the management of patients with prior LAM use, especially in cases of LAM resistance or refractoriness.

The current study has several limitations. First, it is a retrospective study and included patients treated with ETV for at least 30 months. Because we focused on the long-term efficacy and results with ETV therapy, the present study included patients who underwent prolonged follow-up. Therefore, patients who developed ETV resistance before 30 months may be excluded. This could underestimate the incidence of ETV resistance in the study population. However, the cumulative probabilities of ETV resistance in the present study were similar to those of previous studies as mentioned above. ${ }^{8,14}$ Second, the number of patients who experienced LAM was relatively small.

In conclusion, ETV resistance was more frequently developed in LAM-use patients with chronic hepatitis B. However, LAM use without refractoriness did not affect the development of ETV resistance. Therefore, the response to LAM treatment has to be taken into account when considering ETV therapy for the treatment of chronic hepatitis $B$ in patients with prior LAM use. Furthermore, serum HBV DNA level at month 12 of ETV treatment was a major predictor of ETV resistance development. Patients with a higher serum HBV DNA level (>250 IU/mL) after 12 months of ETV therapy should be carefully observed for the development of ETV resistance. 


\section{Acknowledgements}

This study was supported by a grant of the Korean Health Technology R\&D Project, Ministry of Health \& Welfare, Republic of Korea (A121776).

\section{Conflicts of Interest}

No petential conflict of interest relevant to this article is reported.

\section{REFERENCES}

1. Shepard CW, Simard EP, Finelli L, Fiore AE, Bell BP. Hepatitis B virus infection: epidemiology and vaccination. Epidemiol Rev 2006;28:112-125.

2. Fattovich G, Bortolotti F, Donato F. Natural history of chronic hepatitis $B$ : special emphasis on disease progression and prognostic factors. J Hepatol 2008;48:335-352.

3. European Association For The Study Of The Liver. EASL clinical practice guidelines: Management of chronic hepatitis B virus infection. J Hepatol 2012;57:167-185.

4. Hadziyannis SJ, Papatheodoridis GV, Dimou E, Laras A, Papaioannou C. Efficacy of long-term lamivudine monotherapy in patients with hepatitis B e antigen-negative chronic hepatitis B. Hepatology 2000;32:847-851.

5. Leung NW, Lai CL, Chang TT, Guan R, Lee CM, Ng KY, et al. Extended lamivudine treatment in patients with chronic hepatitis $B$ enhances hepatitis $B$ e antigen seroconversion rates: results after 3 years of therapy. Hepatology 2001;33:1527-1532.

6. Lok AS, Lai CL, Leung N, Yao GB, Cui ZY, Schiff ER, et al. Long-term safety of lamivudine treatment in patients with chronic hepatitis $B$. Gastroenterology 2003;125:1714-1722.

7. Lai CL, Shouval D, Lok AS, Chang TT, Cheinquer H, Goodman $Z$, et al. Entecavir versus lamivudine for patients with $\mathrm{HBeAg}$-negative chronic hepatitis B. N Engl J Med 2006;354:1011-1020.

8. Tenney DJ, Rose RE, Baldick CJ, Pokornowski KA, Eggers BJ, Fang $J$, et al. Long-term monitoring shows hepatitis $B$ virus resistance to entecavir in nucleoside-naive patients is rare through 5 years of therapy. Hepatology 2009;49:1503-1514.

9. Fung J, Lai CL, Seto WK, Yuen MF. Nucleoside/nucleotide analogues in the treatment of chronic hepatitis B. J Antimicrob Chemother 2011;66:2715-2725.

10. Tenney DJ, Levine SM, Rose RE, Walsh AW, Weinheimer SP, Discotto $L$, et al. Clinical emergence of entecavir-resistant hepatitis $B$ virus requires additional substitutions in virus already resistant to Lamivudine. Antimicrob Agents Chemother 2004;48:3498-3507.

11. Chang TT, Gish RG, de Man R, Gadano A, Sollano J, Chao YC, et al. A comparison of entecavir and lamivudine for $\mathrm{HBeAg}$-positive chronic hepatitis B. N Engl J Med 2006;354:1001-1010.

12. Reijnders JG, Deterding K, Petersen J, Zoulim F, Santantonio T, Buti $M$, et al. Antiviral effect of entecavir in chronic hepatitis $B$ : influence of prior exposure to nucleos(t)ide analogues. J Hepatol 2010;52:493-500.

13. Ide T, Sata M, Chayama K, Shindo M, Toyota J, Mochida S, et al. Evaluation of long-term entecavir treatment in stable chronic hepatitis B patients switched from lamivudine therapy. Hepatol Int 2010;4:594-600.

14. Lee JH, Cho Y, Lee DH, Lee M, Yoo JJ, Choi WM, et al. Prior exposure to lamivudine increases entecavir resistance risk in chronic hepatitis B Patients without detectable lamivudine resistance. Antimicrob Agents Chemother 2014;58:1730-1737.

15. Ono A, Suzuki F, Kawamura Y, Sezaki H, Hosaka T, Akuta N, et al. Long-term continuous entecavir therapy in nucleos(t)ide-naïve chronic hepatitis B patients. J Hepatol 2012;57:508-514.

16. Suzuki F, Akuta N, Suzuki Y, Yatsuji $H$, Sezaki $H$, Arase $Y$ et al. Efficacy of switching to entecavir monotherapy in Japanese lamivudinepretreated patients. J Gastroenterol Hepatol 2010;25:892-898.

17. Liaw $Y F$, Gane E, Leung N, Zeuzem S, Wang Y, Lai $C L$, et al. 2-Year GLOBE trial results: telbivudine Is superior to lamivudine in patients with chronic hepatitis B. Gastroenterology 2009;136:486-495.

18. Hadziyannis SJ, Tassopoulos NC, Heathcote EJ, Chang TT, Kitis G, Rizzetto $M$, et al. Long-term therapy with adefovir dipivoxil for HBeAg-negative chronic hepatitis B for up to 5 years. Gastroenterology 2006;131:1743-1751.

19. Yuen MF, Sablon E, Hui CK, Yuan HJ, Decraemer H, Lai CL. Factors associated with hepatitis $B$ virus DNA breakthrough in patients receiving prolonged lamivudine therapy. Hepatology 2001;34:785-791.

20. Zeuzem S, Gane E, Liaw YF, Lim SG, DiBisceglie A, Buti M, et al. Baseline characteristics and early on-treatment response predict the outcomes of 2 years of telbivudine treatment of chronic hepatitis B. J Hepatol 2009;51:11-20.

21. Kwon DH, Kim IH, Choung BS, Ahn DS, Yoo SH, Park SB, et al. Continuous long-term entecavir therapy in naive chronic hepatitis $B$ patients showing partial virologic response. Gut Liver 2013;7:712718.

22. Bang SJ, Kim BG, Shin JW, Ju HU, Park BR, Kim MH, et al. Clinical course of patients with insufficient viral suppression during entecavir therapy in genotype C chronic hepatitis B. Dig Liver Dis 2013;45:600-605.

23. Baran B, Soyer OM, Ormeci AC, Gokturk S, Evirgen S, Bozbey HU, et al. Efficacy of tenofovir in patients with Lamivudine failure is not different from that in nucleoside/nucleotide analogue-naive patients with chronic hepatitis B. Antimicrob Agents Chemother 2013;57:1790-1796.

24. Corsa AC, Liu Y, Flaherty JF, Mitchell B, Fung SK, Gane E, et al. No 
resistance to tenofovir disoproxil fumarate through 96 weeks of treatment in patients with lamivudine-resistant chronic hepatitis B. Clin Gastroenterol Hepatol 2014;12:2106-2112.e1.

25. Fung $S$, Kwan $P$, Fabri M, Horban $A$, Pelemis $M$, Hann HW, et al. Ran- domized comparison of tenofovir disoproxil fumarate vs emtricitabine and tenofovir disoproxil fumarate in patients with lamivudine-resistant chronic hepatitis B. Gastroenterology 2014;146:980-988. 